\title{
A unique CD27 - IgD - B-cells population in the sputum of severe eosinophilic asthma associated with airway autoimmunity
}

Nadia Suray Tan ${ }^{1}$, Manali Mukherjee ${ }^{2}$, Sheau Yng Lim ${ }^{1}$, Angeline Rouers ${ }^{3}$, You Yi Hwang $^{3}$, Chung Hwee Thiam ${ }^{1}$, W.S. Daniel Tan ${ }^{4}$, Wupeng Liao ${ }^{4}$, WS Fred Wong ${ }^{4}$, Mei Fong Liew ${ }^{1}$, Parameswaran $\mathrm{Nair}^{2}$, Anis Larbi ${ }^{3}$, De Yun Wang ${ }^{1}$, Katja Fink ${ }^{3}$, Veronique Angeli ${ }^{1}$, and Hui Fang Lim $^{1}$

${ }^{1}$ National University Singapore Yong Loo Lin School of Medicine

${ }^{2}$ McMaster University Faculty of Health Sciences

${ }^{3}$ Singapore Immunology Network

${ }^{4}$ National University of Singapore Department of Pharmacology

February 15, 2022

\section{Allergy: Letter to the Editor}

A unique CD27 $^{-}$IgD $^{-}$B-cells population in the sputum of severe eosinophilic asthma associated with airway autoimmunity

Nadia Suray Tan ${ }^{1 *}$, Manali Mukherjee ${ }^{2 *}$, Sheau Yng Lim ${ }^{3}$, Angeline Rouers ${ }^{4,5}$, You Yi Hwang ${ }^{4}$, Chung Hwee Thiam ${ }^{3}$, W.S. Daniel Tan ${ }^{6}$, Wupeng Liao ${ }^{6}$,W.S. Fred Wong ${ }^{6}$, Mei Fong Liew ${ }^{1,7}$, Parameswaran Nair $^{2}$, Anis Larbi ${ }^{4}$, De Yun Wang ${ }^{8}$, Katja Fink ${ }^{4}$, Veronique Angeli ${ }^{3,9 \#, ~ H u i ~ F a n g ~ L i m ~}{ }^{1,3,7 \#}$

*equal contribution; \# Co-senior authors

${ }^{1}$ Department of Medicine, Yong Loo Lin School of Medicine, National University of Singapore, Singapore.

2 Division of Respirology, Firestone Institute for Respiratory Health, St Joseph's Healthcare, McMaster University, Hamilton, Ontario, Canada

${ }^{3}$ Immunology Translational Research Programme, Department of Microbiology and Immunology, Yong Loo Lin School of Medicine and Immunology Program, Life Science Institute, National University of Singapore, Singapore

${ }^{4}$ Singapore Immunology Network, Agency for Science, Technology and Research A*STAR, Singapore

${ }^{5}$ A*STAR ID Labs, Agency for Science, Technology and Research, Singapore

${ }^{6}$ Department of Pharmacology, Yong Loo Lin School of Medicine, National University of Singapore

${ }^{7}$ Division of Respiratory and Critical Care Medicine, Department of Medicine, National University Hospital, National University Health System, Singapore

8 Department of Otolaryngology, Yong Loo Ling School of Medicine, National University of Singapore, Singapore

${ }^{9}$ Singapore-Hebrew University of Jerusalem Alliance for Research and Enterprise (SHARE), National University of Singapore

Corresponding author(s): 
Hui Fang Lim and Veronique Angeli, 1E Kent Ridge Road, Singapore 119228.

E-mail: hui_fang_lim@nuhs.edu.sg and micva@nus.edu.sg

\section{Short summary:}

A unique CD27- $\mathrm{IgD}^{-}$B-cells population was found to be significantly higher in number and proportion in the sputum of severe eosinophilic asthma, particularly the oral corticosteroid-dependent subgroup and might be related to a local autoimmunity phenomenon.

Key words : severe asthma, B-cells, airway autoimmunity, autoantibodies

Word count :645; Table :0; Figures :2;References : 6

Online supplementary: 3 tables, 1 figure

To the editor:

A unique cross talk between the innate and adaptive cells underlie asthma pathobiology. Within the adaptive domain, T lymphocytes have a clear pathogenic role, while that of B lymphocytes remain lesser known. B-cells support IgE-dependant allergic inflammation, evident from $\mathrm{IgE}^{+}$B-cells in asthmatic airways, postaeroallergen challenge ${ }^{1}$. Moreover, the presence of anti-eosinophil peroxidase (EPX) and anti-nuclear/extranuclear antibodies (ANAs) detectable in sputa and not in the circulation, indicate a B-cell dominant mechanism capable of in situ autoantibody generation ${ }^{2}$. We therefore aimed to detect and characterize B-cell subsets in severe eosinophilic asthma (SEA) and further probe into a possible association with airway autoimmunity.

We recruited stable moderate and severe eosinophilic asthmatics despite adherence to inhaled/oral corticosteroids (ICS/OCS), corresponding to Global Initiative for Asthma (GINA) treatment steps 4-5, and a comparator healthy control (HC) population. All subjects provided written informed consent. Induced sputum and peripheral blood mononuclear cells (PBMC) were collected for immunophenotyping (cohort 1, $\mathrm{n}=36$ ) and subsequent B-cell characterization (cohort 2, $\mathrm{n}=34$ ) (refer toTable E1 and online supplementary for methodology).

Flow cytometry analysis (gating strategy Fig 1A, Table E2) ${ }^{\mathbf{3}}$ showed that out of $\mathrm{CD}^{4} 5^{+}$sputa cells, only the CD3 ${ }^{-} \mathrm{CD} 19^{+}$subset (identified as B-cells) were increased in asthma vs. HC (Fig.1B ), especially in SEA who remained eosinophilic despite ICS/OCS (Fig.1C ). B-cells enumerated from PBMCs remain unremarkable between the subgroups (Fig.E1 ). The B-cell numbers correlated with indices of asthma severity viz., sputum eosinophils $(\mathrm{r}=0.5, P=0.01)$, reduced lung function $(\mathrm{r}=-0.4, P=0.01)$, and cytokines that support B-cell recruitment, activation, differentiation, and survival, such as IL-6 $(\mathrm{r}=-0.3, P=0.05)$ and B-cells activating factor $(\mathrm{BAFF}, \mathrm{r}=-0.3, P=0.04)$ ) (Table E3 $)^{4}$.

In Cohort 2 (gating strategy Fig.1E, Fig.E1E, Table E2)compared to matched PBMC population, naïve B-cells ( $\left.\mathrm{CD}^{-} \mathrm{CD} 19^{+} \mathrm{CD} 27^{-} \mathrm{IgD}{ }^{+}\right)$, classic memory cells $\left(\mathrm{CD} 19^{+} \mathrm{CD} 27^{+} \mathrm{IgD}^{-} \mathrm{CD} 38^{\mathrm{dim} /-}\right)$ and a unique isotype-switched $\mathrm{CD}^{-} \mathrm{CD}^{-}{ }^{+} \mathrm{IgM}^{-} \mathrm{CD} 27^{-} \mathrm{IgD}^{-}$or double-negative (DN) subset were significantly higher in sputa(Fig.1F). Within the sputa, DN B-cells constitute $~ 80 \%$ of the total B-cell population, and the numbers are significantly elevated in SEA (Fig.1G, H ). These cells exhibit both antigen-activated (HLA-DR ${ }^{+} \mathrm{CD}_{40}{ }^{+}$) and immune-exhaustion surface markers $\left(\mathrm{CD} 11 \mathrm{c}^{+} \mathrm{CD} 21^{-}\right)$(Fig.1E , depicts subtyping of the DN B-cells), indicating functional heterogeneity.

DN B-cells are typically extrafollicular and reported in several autoimmune diseases associated with the pathology $y^{4,5}$. These cells are isotype-switched and has the capability of acting as antibody-secreting cells (ASCs) in situ, with/without antigen-stimulation ${ }^{6}$. Given, sputum autoantibodies (localized to the airways) have been reported in a similar SEA population ${ }^{2}$, we investigated (i) sputum ANAs and anti-EPX IgG (as described $^{2}$ ), and (ii) evidence of in situ Immunoglobulin (Ig) isotypes and autoantibody secretion by isolated sputum cells with increased DN B subsets (method online). 
Both ANAs and anti-EPX IgGs were detectable, and the former was significantly higher in the SEA subset, in particular those who were OCS-dependent (Fig.2A, B ). ANAs correlated significantly with the anti-EPX IgG (Fig2.C ) confirming our previous observation ${ }^{2}$, but more importantly with the percentage $(\mathrm{r}=0.3, P$ $=0.03)$ and the number of B-cells $(\mathrm{r}=0.4, P=0.009)$ in the sputa (Fig.2D,E ). Sputum B-cells showed an increased trend in autoimmune airways (Fig.2F ). Finally, ELISpot (cohort 2) confirmed that sputum cells that comprise of high percentage of DN B-cells, derived from moderate-severe eosinophilic asthmatics are capable of producing the key mucosal Ig subtype IgA, as well as IgGex vivo, in addition to specific Igs reactive to the coated autoantigen, EPX (Fig.2G-I ).

We acknowledge that the study is limited by its small sample size; HC are not age-matched, mainly due to increasing co-morbidities with age that precludes recruitment, and also inadequate sputum production. Detecting mechanisms of local class-switch of IgA to IgG autoantibodies were also beyond the immediate scope of the study. Nevertheless, we report an increased B-cells with a unique population of DN B-cells in SEA airways at steady state (i.e., no exacerbation), that can spontaneously secrete Igs, including autoreactive-Igs, without antigen stimulation. This warrants a longitudinal study to examine the role of DN B-cells in asthma, their functional relevance at exacerbations, in situ autoantibody generation and development/maintenance of airway autoimmunity over time.

Ethical approval of the project was obtained from the National Healthcare Group Domain Specific Review Board (Ref:2018/00910). All subjects provided written informed consent.

Author contributions: H.F.L. and V.A. conceived the idea and takes responsibility for overall guarantee of the paper. N.S.T and M.M. developed the experimental design. S.Y.L. and C.H.T. were involved in flow cytometry and protein assay analysis. A.R. and K.F. were involved in development of ELISpot. Y.Y.H. and A.L. were involved in the planning and acquisition of multiparametric flow cytometry panel. W.S.D.T., W.P. and W.S.F.W. performed multiplex assay and PBMC processing. N.S.T., M.F.L. and H.F.L. were involved in patient care, recruitment and coordination. M.M., P.N., D.Y.W. were involved in scientific advice. H.F.L., V.A. and N.S.T. analyzed data. All authors have read and consented to current manuscript draft.

Funding: The study was supported by grants from the National Research Foundation, under its Campus for Research Excellence and Technological Enterprise (CREATE) and National Medical Research Council (Project ID: MOH-000146-01).

\section{References:}

1. Oliveria JP, Salter BM, MacLean J, Kotwal S, Smith A, Harris JM, et al. Increased IgE+ B Cells in Sputum, but Not Blood, Bone Marrow, or Tonsils, after Inhaled Allergen Challenge in Subjects with Asthma. Am J Respir Crit Care Med 2017; 196:107-9.

2. Mukherjee M, Bulir DC, Radford K, Kjarsgaard M, Huang CM, Jacobsen EA, et al. Sputum autoantibodies in patients with severe eosinophilic asthma. J Allergy Clin Immunol 2018; 141:1269-79.

3. Jenks SA, Cashman KS, Zumaquero E, Marigorta UM, Patel AV, Wang X, et al. Distinct Effector B Cells Induced by Unregulated Toll-like Receptor 7 Contribute to Pathogenic Responses in Systemic Lupus Erythematosus. Immunity 2018; 49:725-39 e6.

4. Bernard NJ. Double-negative B cells. Nature Reviews Rheumatology 2018; 14:684-.

5. Ma S, Wang C, Mao X, Hao Y. B Cell Dysfunction Associated With Aging and Autoimmune Diseases. Frontiers in Immunology 2019; 10.

6. Min Q, Meng X, Zhou Q, Wang Y, Li Y, Lai N, et al. RAG1 splicing mutation causes enhanced B cell differentiation and autoantibody production. JCI Insight 2021; 6 . 


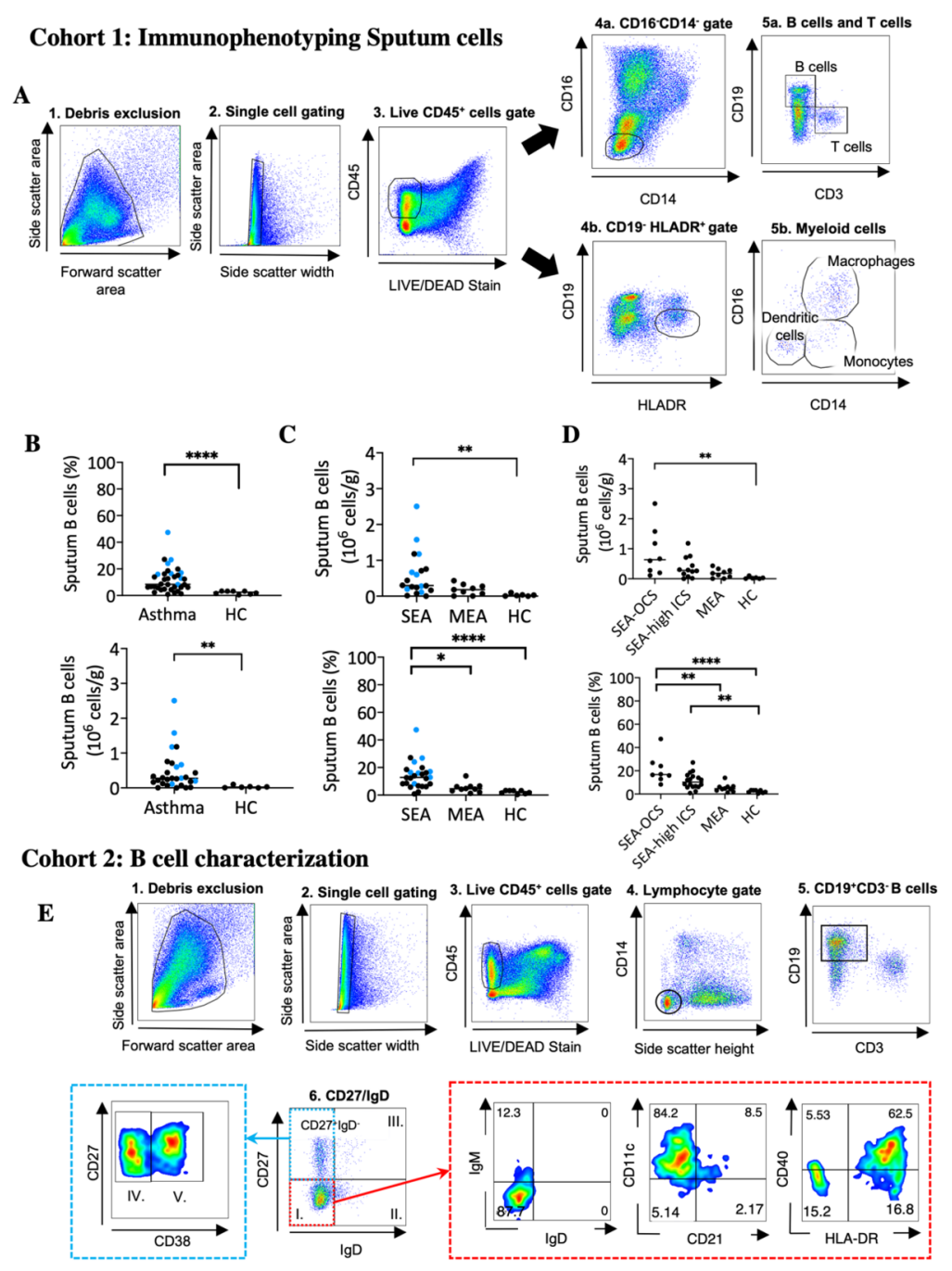

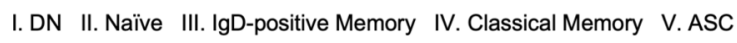

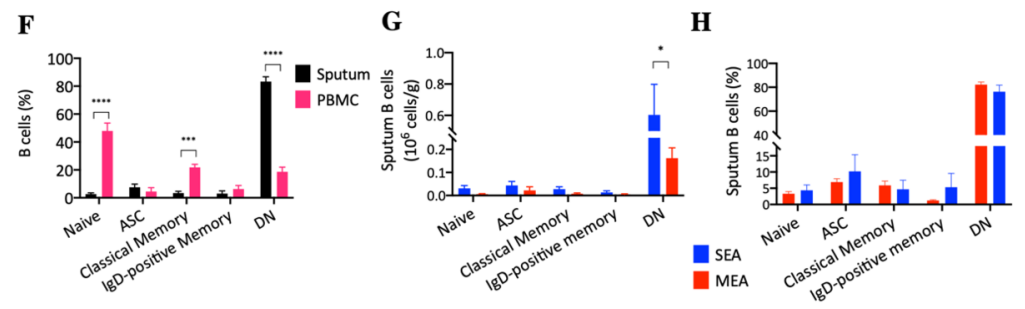

Figure 1. Comparison of sputum and PBMC B-cells populations: I. Top panel: Cohort 1: (A) Gating strategy for immunephenotyping CD45+ cells in sputa. Total and percent B-cells in sputum is plotted stratified by $(\mathbf{B})$ asthma vs healthy, $\mathrm{HC}, \mathrm{n}=8,(\mathbf{C})$ moderate (MEA, $\mathrm{n}=10)$ vs severe eosinophilic asthma (SEA, $n=26$ ) (blue symbols indicate those on oral corticosteroid, OCS; (D)OCS-dependent SEA ( $\mathrm{n}=8$ ), ICSdependent SEA ( $\mathrm{n}=18)$, MEA and HC. Kruskal-Wallis and Dunn's multiple comparison tests. II. Bottom panel Cohort 2: (E) Gating strategy for multiparameter analysis of B-cells subsets. of double negative (DN) CD27-IgD- B-cells of surface expression of IgM, CD11C, CD21, CD40 and HLA-DR, in addition to naïve, classical memory and antigen secreting cells (ASCs). (F)B-cells subtype in sputa vs PBMC. The total 
number and proportion of B cell subsets are plotted in $(\mathbf{G}, \mathbf{H})$ compared between moderate eosinophilic asthma (MEA) and severe eosinophilic asthma (SEA). 2-way ANOVA with the Sidak's multiple comparisons test were used for comparison of B-cell subtypes; ${ }^{*} \mathrm{P}[?] 0.05,{ }^{* *} \mathrm{P}[?] 0.01,{ }^{* * * *} \mathrm{P}[?] 0.0001$.

A

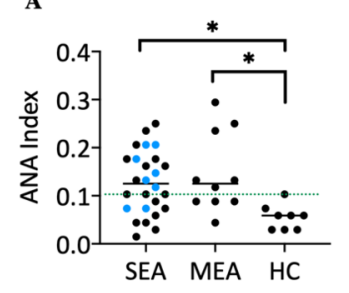

D

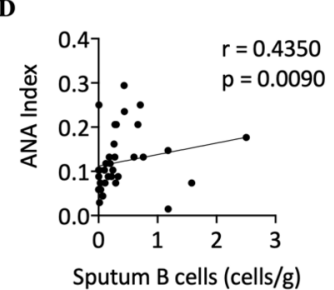

G

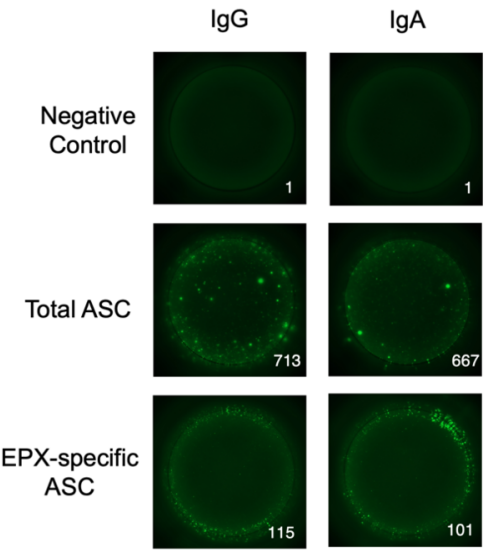

C
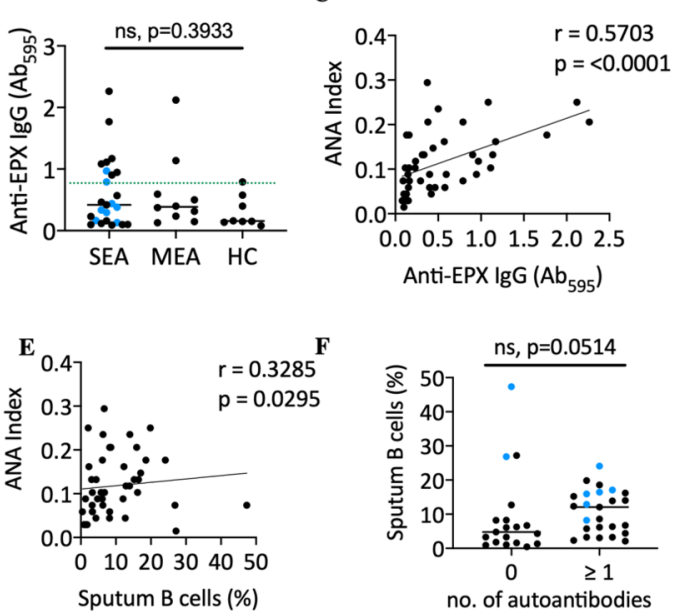

H

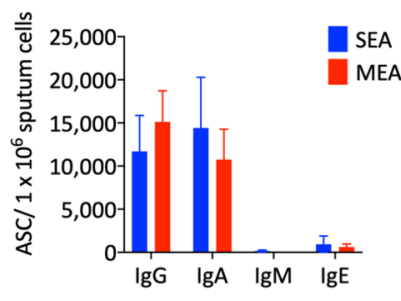

I

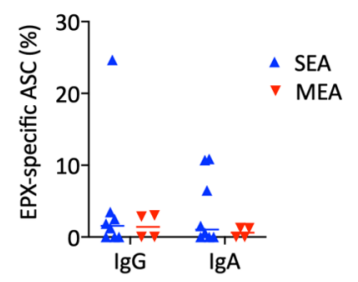

Figure 2. Autoantibody profile and fuctional analysis of sputum B-cells. (A, B) Significantly higher anti-nuclear antibody (ANA) was observed in SEA and MEA as compared to HC. The calculated cut-off was based on $95^{\text {th }}$ percentile $\mathrm{HC}$ subjects, as represented by dotted lines. (C) Significant positive correlation between sputum ANA and anti-EPX IgG. (D, E) Sputum ANA is positively correlated with sputum B-cell proportion and numbers. (F) Sputum B-cells showed an increased trend in autoimmune airways $(\mathbf{G}, \mathbf{H})$ Profile of unstimulated B-cells ELISpot assay in SEA $(\mathrm{n}=17)$ and MEA $(\mathrm{n}=5)$ showed predominant IgG and IgA ASC. (I) ELISpot assay detects antibody-secreting cells in sputa, that produce anti-EPX IgG and IgA 